\title{
Kinetics of the laser-induced solid phase crystallization of amorphous silicon - Time-resolved Raman spectroscopy and computer simulations
}

\author{
J. Očenášek, ${ }^{1, a)}$ P. Novák, ${ }^{1}$ and L. Prušáková ${ }^{1}$ \\ ${ }^{1}$ New Technologies - Research Centre, University of West Bohemia, 30614, Plzeň, Czech Republic
}

This study demonstrates that a laser-induced crystallization instrumented with Raman spectroscopy is, in general, an effective tool to study the thermally activated crystallization kinetics. It is shown, for the solid phase crystallization of an amorphous silicon thin film, that the integral intensity of Raman spectra corresponding to the crystalline phase grows linearly in the time-logarithmic scale. A mathematical model, which assumes random nucleation and crystal growth, was designed to simulate the crystallization process in the non-uniform temperature field induced by laser. The model is based on solving the Eikonal equation and the Arhenius temperature dependence of the crystal nucleation and the growth rate. These computer simulations successfully approximate the crystallization process kinetics and suggest that laser-induced crystallization is primarily thermally activated.

(Submitted to Applied Surface Science on September 2016)

\footnotetext{
a) Electronic mail: ocenasek@ntc.zcu.cz
} 


\section{Introduction}

The laser-induced crystallization (LIC) of hydrogenated amorphous silicon (a-Si:H) has been a subject of extensive research in recent years, motivated predominantly by technological applications to thin film semiconductors. LIC studies instrumented by Raman spectroscopy appeared more recently [1-5], enabling in situ quantification of the phase composition, along with an indirect estimate of the sample temperature and other structural properties $[6,7]$. The main advantage of this approach is that the same laser may simultaneously control the LIC process and also serve as the Raman excitation laser source. Laser as a heat source, in comparison to conventional furnace heating, also offers fast heatingup of the probe to very high temperatures. Raman instrumented LIC is thus a simple and effective analytical technique to study the phase transformation kinetics at high temperatures.

This study examines the solid phase crystallization of thin film amorphous silicon (a-Si) induced by a continuous-wave laser. The progress of crystallization is monitored by sequential Raman spectroscopy. For closer analysis by means of computation simulations, a mathematical model of the crystallization process was designed, with the capability to account for the existing non-uniform temperature field.

\section{Experimental part}

\subsection{Sample preparation and experimental setup}

A film of a-Si:H was deposited on a corning glass substrate using plasma-enhanced chemical vapor deposition from silane and argon gas mixture precursors (10\% of SiH4 in $\mathrm{Ar}$ ). The hydrogen content in the film was $13 \%$, as estimated by comparing Raman scattering intensity related to the vibration of $\mathrm{Si}-\mathrm{H}_{2}$ and $\mathrm{Si}-\mathrm{H}$ bonds with the intensity of $\mathrm{Si}-\mathrm{Si}$ bonds [8]. The film thickness for all experiments was $1000 \mathrm{~nm}$, which ensures a very limited effect of the substrate heat conductivity on the sample heating by the laser [9]. For the Raman scattering experiments, a DXR Raman microscope was used with a solid state Nd:YVO4 $532 \mathrm{~nm}$ laser source, with the beam quality factor $\mathrm{M}^{2}<1.3$, focused to a spot with Gaussian radius $550 \mathrm{~nm}$. 
Raman measurements were performed as follows. The Raman laser source was turned on and the sample was continuously irradiated for $400 \mathrm{~s}$. Within this period, separate Raman spectra with a short acquisition time (2s) were recorded repetitively, in order to monitor gradual phase transformation in the sample. Experiments using four settings of laser power $-7.0,7.5,8.5$ and $10 \mathrm{~mW}$ - were performed. Additional measurements using powers lower than $7 \mathrm{~mW}$ did not show any marks of crystallization.

Fig. 1 shows spectral lines recorded at selected times with the progressive appearance of the crystalline silicon (c-Si) transverse optical (TO) mode, proving the presence of the crystallization. Note that there may be a significant difference between the spectra acquired by a long-time measurement and short-time acquisition (see the dashed line in Fig. 1), since the material transforms progressively. A more detailed study, performed with identical a-Si samples, of the thermally induced c-Si peak broadening and position shift related to the LIC process, was published elsewhere [9].

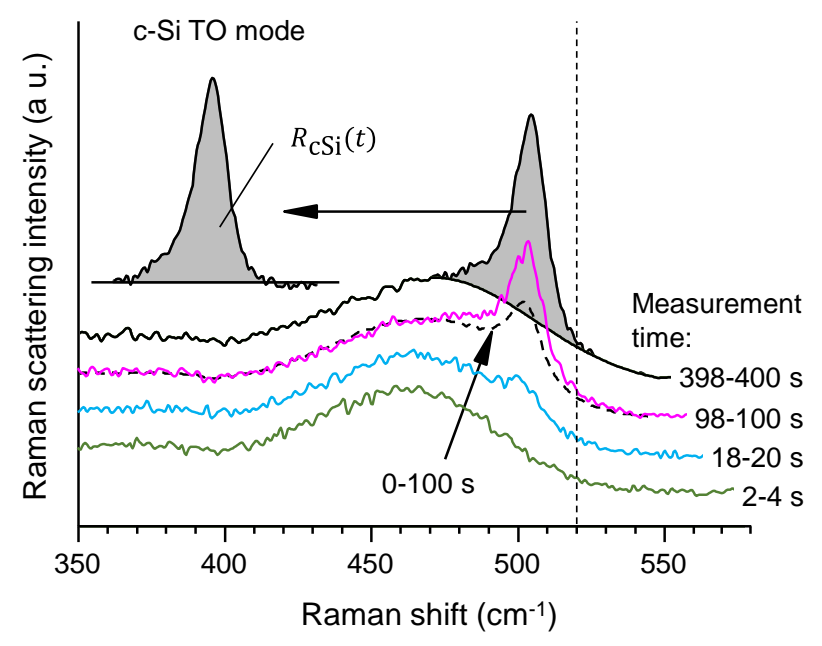

FIG. 1. Selected Raman spectra recorded in $2 \mathrm{~s}$ periods at different times (laser power $8.5 \mathrm{~mW}$ ). Reference c-Si TO mode peak position $520 \mathrm{~cm}^{-1}$ is indicated. The dashed spectrum marks a longtime spectra acquisition. Note that lines were vertically shifted for better clarity. The inset shows extracted c-Si TO mode. 


\subsection{Experimental results}

To analyze the crystallization kinetics, the c-Si TO scattering rate (marked $R_{\mathrm{cSi}}$ ) was quantified for each of the spectral lines. See Fig. 2 showing three experiments for each laser power. The $R_{\mathrm{cSi}}$ was calculated as an integral intensity given by the area of the c-Si TO mode peak, where the peaks were obtained by subtracting the a-Si and the background signal from the spectral line (see the insert in Fig. 1). We observe that the scattering rate evolution $R_{\mathrm{cSi}}(t)$ significantly depends on the applied laser power and, interestingly, grows proportionally to the time logarithm with a slope invariant to the laser power. Note that for the case with power $7 \mathrm{~mW}$, in two of the three experiments no crystallization was detected, which may be related to the material inhomogeneity. Further analyses using computational simulations are given later.

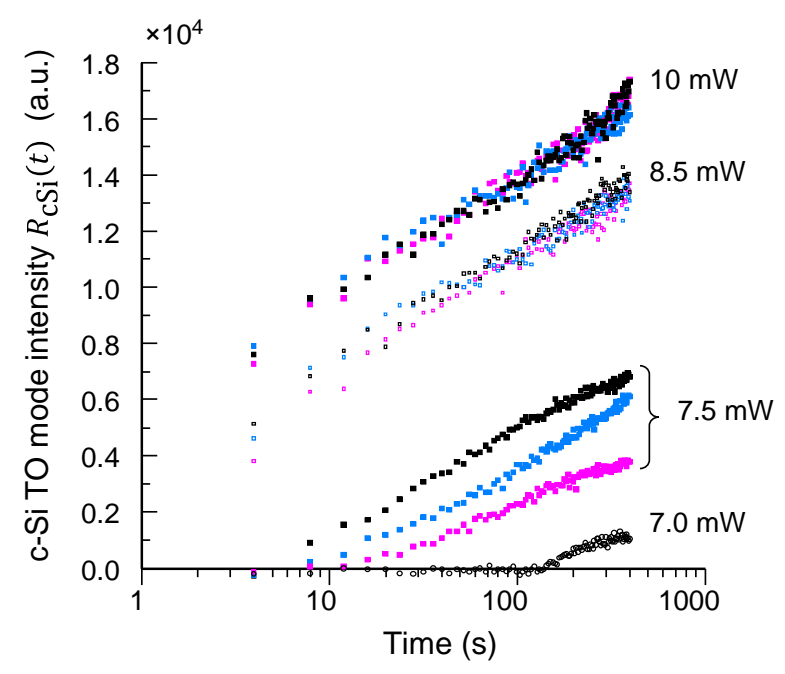

FIG. 2. Time evolution of the $\mathrm{c}-\mathrm{Si}$ scattering rate $R_{\mathrm{cSi}}(t)$ measured experimentally; three experimental sets are given for each laser power.

Optical microscope photos in Fig. 3 show gradual growth of the crystalline zone with increasing irradiation time and the influence of the laser power. It was demonstrated [9] that the inner darker zone marks the crystallized volume, whereas the bright area corresponds to a decreased hydrogen content caused by thermally-driven hydrogen diffusion. As a-Si and c-Si phases have different optical 
properties, the crystalline phase can be visualized with light microscopy [10], although with somewhat limited resolution. On the contrary, for the laser focus and powers applied in this study, AFM or SEM microscopy cannot reveal any changes related to the laser irradiation [9], indicating that the laserinduced temperatures are below the melting point. Note that clear marks (SEM detectable) are left on the sample surface when the Si melting temperature $\left(1414^{\circ} \mathrm{C}\right)$ is reached $[11,12]$.

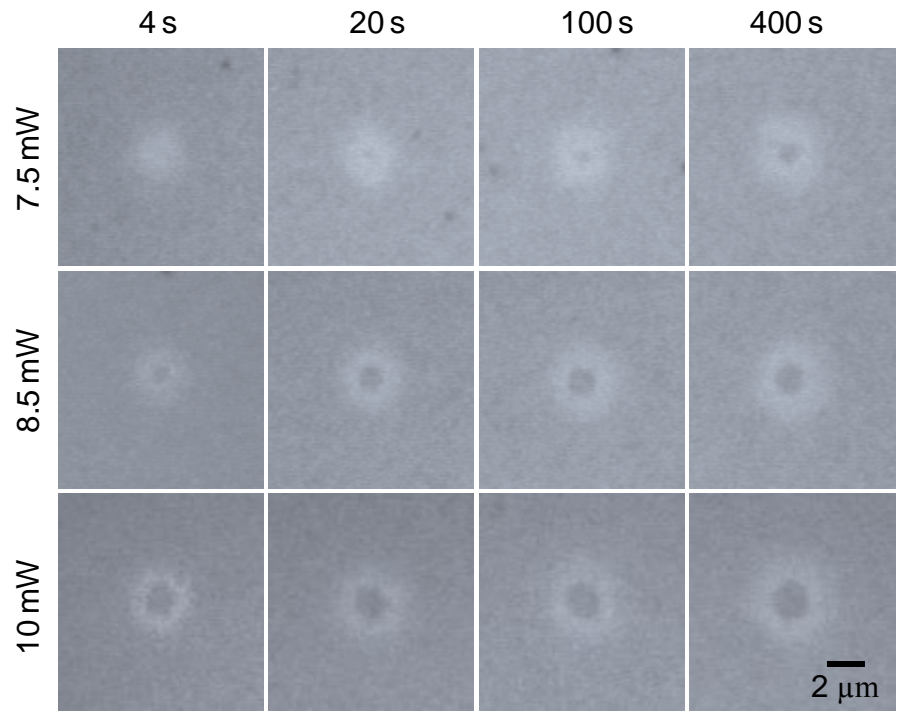

FIG. 3. Surface spots captured by light microscopy for different laser power and irradiation time. The bright area corresponds to a decreased hydrogen content, the inner darker zone marks the crystallized volume.

\section{Computer simulations}

\subsection{Modeling method}

A numerical simulation of the crystallization process was designed to demonstrate that a random crystallite nucleation and the crystal growth together with the Arrhenius law approximates well the crystallization kinetics. The laser-induced temperature field was calculated by means of an analytical formula, where a volumetric heat source was assumed $[13,14]$. Only the steady state 
temperature is considered, since the thermal diffusivity is such that the transient temperature field is stabilized in a time fraction less than $10^{-3} \mathrm{~s}[9]$.

The nucleation rate $N$ and the growth velocity $G$ following the Arrhenius equation are

$$
\begin{aligned}
& N=N_{0} \exp \left(-E_{\mathrm{N}} / k_{\mathrm{B}} \theta\right), \\
& G=G_{0} \exp \left(-E_{\mathrm{G}} / k_{\mathrm{B}} \theta\right),
\end{aligned}
$$

where $E_{\mathrm{N}}$ and $E_{\mathrm{G}}$ are activation energies of the nucleation and the growth, respectively, $k_{B}$ denotes the Boltzmann constant and $\theta$ is the temperature. $N_{0}$ and $G_{0}$ are material parameters. The numerical simulation is based on a random generation of grain nuclei at a rate given by eq. (1). The so-called arrival function $T(x)$, which estimates the time when the nucleated grain grows to a point $x$, is then calculated for each grain. In the case of a non-uniform growth rate, as given by (2), the problem leads to Eikonal's equation, which is a boundary value problem defined in [15] as

$$
\left|\nabla T_{i}\right| G=1, T=\tau_{i} \text { on } \xi_{i},
$$

where $G(x)$ is the growth rate, $T_{i}(x)$ is the arrival function of a grain $i$ nucleating at position $\xi_{i}$ and time $\tau_{i}$. Since a three-dimensional problem is being solved, points $x$ and $\xi_{i}$ are position vectors with three components. The scalar filed $T_{i}(x)$ - obtained by solving Eq. (3) - specifies the time when the boundary of the grain $i$ reaches the material point $x$. For the case of multiple grains, a selected material point (at position $x$ ) undergoes phase transformation at the first moment - when the boundary of any present grain arrives. This defines the total arrival function $\mathbb{T}(x)$, expressed by means of the minimum as

$$
\mathbb{T}(x)=\min _{i}\left\{T_{i}(x)\right\}
$$

Indices $i$ of the minimum value (argument of the minimum) then distinguish individual grains in the crystallized volume, allowing the grain coloring in Figs. 4 and 5.

Finally, the volume $V$ of material crystallized by the time $t$ as 


$$
V(t) \equiv\{x, \mathbb{T}(x) \leq t\}
$$

Equation (3) was numerically solved by means of the Fast Marching Method [15]. The present algorithm was parallelized in terms of simultaneous calculation of the arrival functions $T_{i}$, whose solutions are independent of each other.

\subsection{Model parameters}

The following parameters were used in the numerical simulation. The initial sample temperature was $20^{\circ} \mathrm{C}$. Sample reflectivity 0.4 , corresponding to refraction coefficient $n=4$, was used to account for the partial laser reflection at the sample surface. Absorption coefficient $\alpha=8 \mu \mathrm{m}^{-1}$, together with the laser diameter, defines the volumetric heat source [13]. The thermal conductivity was set to k=3.5 WK-1m-1 in accordance with recent measurements of Braun et al. [16]. Activation energies for the nucleation $E_{\mathrm{N}}=4.2 \mathrm{eV}$ and the growth $E_{\mathrm{G}}=2.6 \mathrm{eV}$ were adopted from the work of Mahan et al. [17].

The rate constants $N_{0}$ and $G_{0}$, present in eqs. (1) and (2), were estimated from the nucleation and growth rates at reference temperature $600^{\circ} \mathrm{C}$, which were $N_{600^{\circ} \mathrm{C}}=4.2 \mu \mathrm{m}^{-3} \mathrm{~min}^{-1}$ and $G_{600^{\circ} \mathrm{C}}=$ $0.7 \mathrm{~nm} \mathrm{~s}^{-1}$. These parameters correspond to crystallization period $\sim 5$ hours at temperature $600^{\circ} \mathrm{C}$ reported in several studies $[6,18-20]$. Since higher nucleation rate results in finer crystallites, the nucleation rate used in this model was adjusted to obtain crystallite size approximately $50 \mathrm{~nm}$, as indicated by the broadening of our Raman spectra or as estimated from the XRD correlation length in the literature [21].

\subsection{Simulation results}

Fig. 4 shows crystallization progress in time and typical polycrystalline structure resulting from the simulation, while Fig. 5 shows the influence of the laser power. The non-isothermal temperature field causes the crystallization process to proceed in two distinct stages, where the initial stage is characterized by multiple nucleation of isolated grains. Later, as the grains grow, they promptly merge 
in one polycrystalline cluster and the crystallization process changes. At locations more distant from the spot center, where there is a lower temperature, the crystal growth and the nucleation rate both decrease significantly. The nucleation rate decrease, together with the presence of the polycrystalline cluster surface, cause the crystal growth to become (although slower) dominant over the nucleation rare. This results in crystal shapes elongated in a radial direction located at the spot periphery (see the video linked with Fig. 4). Comparable morphology was experimentally described after electron beam heating [22].

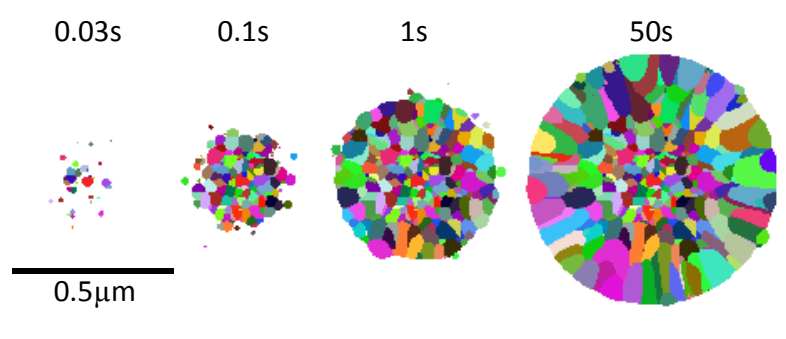

Fig. 4. Numerical simulation of the progress of crystallization for laser power $10 \mathrm{~mW}$, view of the sample surface (Multimedia view)

Note that $E_{\mathrm{N}}>E_{\mathrm{G}}$ was also measured in the work of Köster [22] and, as applied in this model, causes formation of smaller grains at higher temperatures (Fig. 5). The effect of the temperature on the grain size was also observed experimentally [18].

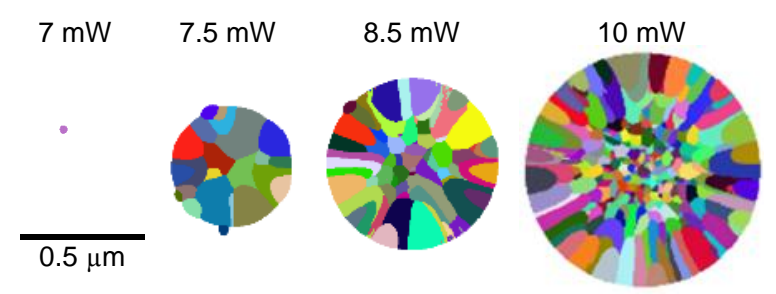

Fig. 5. Surface spots calculated by the model after $400 \mathrm{~s}$ laser irradiation (sample surface view).

The intensity of the Raman excitation laser has a Gaussian profile irradiating the growing crystalline volume, which is the main reason why the scattering rate $R_{\mathrm{cSi}}(t)$ is not directly proportional 
to the crystalline volume. To compare simulation results with the experimental data, the following weight function was used:

$$
w(r, z)=P_{\mathrm{G}}(r) \exp (-2 \alpha z)\left(n_{0}(\omega)+1\right)
$$

which accounts for the Gaussian laser intensity profile $P_{\mathrm{G}}(r)$, exponential damping of the laser source traveling to the depth $z$ and the scattering light traveling the same distance back to the surface and the temperature dependent phonon density function of the Stokes scattering, where $n_{0}(\omega)=$ $\left(\exp \left(\hbar \omega / k_{\mathrm{B}} \theta\right)-1\right)^{-1}$ and $\hbar$ is reduced Planck's constant; $r$ and $z$ are cylindrical coordinates with the origin located at the laser spot center. Then the simulated scattering rate $\tilde{R}_{\mathrm{cSi}}$ may be approximated as a weighted sum of the crystalline volume, i.e.

$$
\tilde{R}_{\mathrm{cSi}}(t)=\int_{V_{\mathrm{cSi}}(t)} w(r, y) d V
$$

See Fig. 2 and 6 to compare the experimental scattering rate $R_{\mathrm{cSi}}(t)$ and the scattering rate $\tilde{R}_{\mathrm{cSi}}(t)$ resulting from the numerical model. Both the simulation and the experiment exhibit after the onset of the crystallization a nearly linear trend in the time-logarithmic scale. The linear trend is thus a consequence of the specific temperature field shape (produced by the laser) and the Arrhenius law. The simulation performed also demonstrated (not shown here) that the $\tilde{R}_{\mathrm{cSi}}(t)$ slope changes with the activation energies $E_{\mathrm{N}}$ and $E_{\mathrm{G}}$.

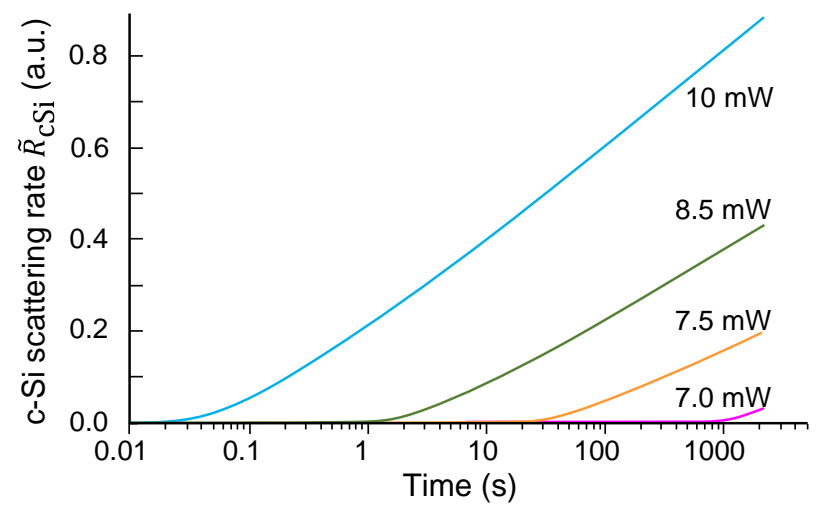

Fig. 6 . The c-Si scattering rate $\widetilde{R}_{\mathrm{cSi}}(t)$ predicted by means of the numerical simulation using eq. (7) 
The temperature values calculated in the model were verified against an indirect temperature measurement using the c-Si peak position shift [23]. The peak position shift was determined by means of a reference measurement with a low laser power $(0.2 \mathrm{~mW})$ after the LIC process, so that the peak shift caused by structural parameters is subtracted. The maximum temperatures at the spot center predicted by the model are $622,708,800$ and $938^{\circ} \mathrm{C}$ for laser powers $7.0,7.5,8.5$ and $10.0 \mathrm{~mW}$, respectively. These values correspond well to the observed thermal c-Si peak position shift, when a correction from the Raman effective temperature is applied [14].

\section{Discussion}

The mathematical model presented can be considered a good approximation; nevertheless, it must be mentioned that the nature of the process is more complex. It is known that also mechanical stress (caused by the deposition or the heating process) in the a-Si:H changes the nucleation and growth rates [17]. In addition, the c-Si phase in comparison to the a-Si phase has a lower absorption coefficient, a smaller index of refraction and higher thermal conductivity [24]. The crystallization process therefore results in changes in the sample reflectance and creates overall a more complicated optical state with additional optical interfaces. Further, additional heat is produced due to the latent heat of the phase transformation, which may lead to an avalanche-like crystallization [25]. All that is coupled with changes in the temperature field which functions as a feedback in the crystallization process. Moreover, nearly all parameters exhibit some temperature dependence, including the Raman cross-section and the heat transfer coefficient. Possible size-effects [26] related to the very limited heated volume are also ignored. A more precise numerical simulation would therefore require solving a strongly nonlinear transient problem coupling crystallization, optics, heat transfer and mechanics. In particular, it is assumed that the $R_{\mathrm{cSi}}(t)$ evolutions for $10 \mathrm{~mW}$ and $8.5 \mathrm{~mW}$ are closer to each other (Fig. 2) than those computationally predicted (Fig. 6), because the crystalline phase has significantly higher thermal conductivity and nearly ten times lower absorption, where both would lead to lower 
temperature than calculated by the model. Above-mentioned may also explain the smaller size of the simulated crystalline cluster than that experimentally observed with the microscope.

\section{Conclusion}

The standard (furnace) isothermal crystallization process, described by the Johnson-MehlAvrami-Kolmogorov model, is characterized by an exponential s-shaped curve specifying the transformed volume fraction in time. On the contrary, a crystallization induced by a focused laser beam is non-isothermal and the heated volume is limited. This study shows that in the laser-induced process, the Raman scattering intensity of the transformed volume grows approximately linearly in the timelogarithmic scale. In summary, this study has shown that:

(i) For the laser-induced crystallization process, the irradiation time has the same significance as in conventional furnace crystallization experiments.

(ii) Ignoring time dependence of the crystallization process may lead to misinterpretation of the measured time-averaged Raman spectra, since the spectra may combine signals from the amorphous and partially crystallized phase in an unknown proportion.

(iii) These experiments, together with numerical simulations, demonstrate that the kinetics of the laser-induced crystallization process is primarily controlled by random nucleation and crystal growth, whose temperature dependence is given by the Arrhenius law.

(iv) The previous point indicates that Raman-instrumented laser-induced crystallization experiments may be used to study its kinetics. And - for instance - a method to identify the activation energy of the process could be developed.

This research was supported by the Czech Ministry of Education, Youth and Sports projects CENTEM CZ.1.05/2.1.00/03.0088 and CENTEM PLUS LO1402. 
[1] D. Munoz-Martin, Y. Chen, M. Morales, J.J. García-Ballesteros, J. Cárabe, J.J. Gandía, J.D. Santos, M. Losurdo, G. Bruno, C. Molpeceres, Parameterization of a-Si crystallization by continuouswave green laser irradiation: from single spot to large area, J. Photonics Energy. 5 (2015) 053086-053086. doi:10.1117/1.JPE.5.053086.

[2] Y.-J. Lee, J.-D. Kwon, D.-H. Kim, K.-S. Nam, Y. Jeong, S.-H. Kwon, S.-G. Park, Structural characterization of wavelength-dependent Raman scattering and laser-induced crystallization of silicon thin films, Thin Solid Films. 542 (2013) 388-392. doi:10.1016/j.tsf.2013.06.055.

[3] W. Wang, J. Huang, Y. Lu, Y. Yang, W. Song, R. Tan, S. Dai, J. Zhou, In situ micro-Raman spectroscopic study of laser-induced crystallization of amorphous silicon thin films on aluminum-doped zinc oxide substrate, J. Mater. Sci. Mater. Electron. 23 (2012) 1300-1305. doi:10.1007/s10854-011-0588-2.

[4] T. Mchedlidze, T. Arguirov, S. Kouteva-Arguirova, M. Kittler, Light induced solid-phase crystallization of Si nanolayers in Si/SiO2 multiple quantum wells, J. Appl. Phys. 107 (2010) 124302. doi:http://dx.doi.org/10.1063/1.3446831.

[5] Y.L. Khait, R. Beserman, A. Chack, R. Weil, W. Beyer, Kinetics of laser-induced low-temperature crystallization of amorphous silicon, Appl. Phys. Lett. 81 (2002) 3347-3349. doi:10.1063/1.1516875.

[6] K. Sharma, M.A. Verheijen, M.C.M. Van De Sanden, M. Creatore, In situ crystallization kinetics studies of plasma-deposited, hydrogenated amorphous silicon layers, J. Appl. Phys. 111 (2012). doi:10.1063/1.3681185.

[7] M. Gan, V. Tomar, An in situ platform for the investigation of Raman shift in micro-scale silicon structures as a function of mechanical stress and temperature increase, Rev. Sci. Instrum. 85 (2014) 13902. doi:http://dx.doi.org/10.1063/1.4861201.

[8] V.A. Volodin, D.I. Koshelev, Quantitative analysis of hydrogen in amorphous silicon using Raman scattering spectroscopy, J. Raman Spectrosc. 44 (2013) 1760-1764. doi:10.1002/jrs.4408.

[9] P. Novák, J. Očenášek, L. Prušáková, V. Vavruňková, J. Savková, J. Rezek, Influence of heat generated by a Raman excitation laser on the structural analysis of thin amorphous silicon film, Appl. Surf. Sci. 364 (2016) 302-307. doi:http://dx.doi.org/10.1016/j.apsusc.2015.12.091.

[10] A.H. Mahan, M.S. Dabney, R.C. Reedy Jr., D. Molina, D.S. Ginley, The use of optical microscopy to examine crystallite nucleation and growth in thermally annealed plasma enhanced chemical vapor deposition and hot wire chemical vapor deposition a-Si:H films, J. Appl. Phys. 111 (2012). doi:10.1063/1.4712045.

[11] P. Alpuim, M.F. Cerqueira, V. Iglesias, G. Machado, J. Borme, Laser patterning of amorphous silicon thin films deposited on flexible and rigid substrates, Phys. Status Solidi Appl. Mater. Sci. (2016). doi:10.1002/pssa.201532980.

[12] F. Delachat, F. Antoni, A. Slaoui, C. Cayron, C. Ducros, J.-F. Lerat, T. Emeraud, R. Negru, K. Huet, P.-L. Reydet, Excimer laser crystallization of amorphous silicon on metallic substrate, Appl. Phys. Mater. Sci. Process. 111 (2013) 807-812. doi:10.1007/s00339-013-7643-1.

[13] M. Lax, Temperature rise induced by a laser beam, J. Appl. Phys. 48 (1977) 3919-3924. doi:http://dx.doi.org/10.1063/1.324265.

[14] J. Očenášek, J. Voldřich, Raman thermometry: Effective temperature of the nonuniform temperature field induced by a Gaussian laser, J. Appl. Phys. 118 (2015). doi:10.1063/1.4937904.

[15] J.A. Sethian, Level Set Methods: Evolving Interfaces in Computational Geometry, Fluid Mechanics, Computer Vision, and Materials Science, Cambridge University Press, 1996. https://books.google.cz/books?id=A-BiQgAACAAJ.

[16] J.L. Braun, C.H. Baker, A. Giri, M. Elahi, K. Artyushkova, T.E. Beechem, P.M. Norris, Z.C. Leseman, J.T. Gaskins, P.E. Hopkins, Size effects on the thermal conductivity of amorphous silicon thin films, Phys. Rev. B - Condens. Matter Mater. Phys. 93 (2016). doi:10.1103/PhysRevB.93.140201. 
[17] A.H. Mahan, M.S. Dabney, D. Molina Piper, W. Nemeth, The effect of film tensile stress on crystallite nucleation and growth in thermally annealed a-Si:H, J. Appl. Phys. 115 (2014). doi:10.1063/1.4865943.

[18] W.-E. Hong, J.-S. Ro, Kinetics of solid phase crystallization of amorphous silicon analyzed by Raman spectroscopy, J. Appl. Phys. 114 (2013). doi:10.1063/1.4818949.

[19] Y. Tao, S. Varlamov, G. Jin, M. Wolf, R. Egan, Effects of annealing temperature on crystallisation kinetics and properties of polycrystalline $\mathrm{Si}$ thin films and solar cells on glass fabricated by plasma enhanced chemical vapour deposition, Thin Solid Films. 520 (2011) 543-549. doi:10.1016/j.tsf.2011.06.089.

[20] M.S. Dabney, P.A. Parilla, L.M. Gedvilas, A.H. Mahan, D.S. Ginley, Altering the nucleation of thermally annealed hydrogenated amorphous silicon with laser processing, Appl. Phys. Lett. 95 (2009). doi:10.1063/1.3259654.

[21] A.H. Mahan, S.P. Ahrenkiel, R.E.I. Schropp, H. Li, D.S. Ginley, A comparison of grain nucleation and grain growth during crystallization of HWCVD and PECVD a-Si:H films, Thin Solid Films. 516 (2008) 529-532. doi:10.1016/j.tsf.2007.06.036.

[22] U. Koster U. ste., Crystallization of amorphous silicon films, Phys. Status Solidi A. 48 (1978) 313321. doi:10.1002/pssa.2210480207.

[23] M. Balkanski, R.F. Wallis, E. Haro, Anharmonic effects in light scattering due to optical phonons in silicon, Phys Rev B. 28 (1983) 1928-1934. doi:10.1103/PhysRevB.28.1928.

[24] D. Han, G. Yue, J.D. Lorentzen, J. Lin, H. Habuchi, Q. Wang, Optical and electronic properties of microcrystalline silicon as a function of microcrystallinity, J. Appl. Phys. 87 (2000) 1882-1888.

[25] P. Lengsfeld, N.H. Nickel, W. Fuhs, Step-by-step excimer laser induced crystallization of a-Si:H, Appl. Phys. Lett. 76 (2000) 1680-1682.

[26] J. Očenášek, P. Novák, S. Agbo, Finite-thickness effect on crystallization kinetics in thin films and its adaptation in the Johnson-Mehl-Avrami-Kolmogorov model, J. Appl. Phys. 115 (2014). doi:10.1063/1.4862858. 